Report to the 14th International Symposium on Spin Physics

October 16-21, 2000, RCNP, Osaka University, Osaka, Japan

\title{
Physics Beyond SM at RHIC with Polarized Protons
}

\author{
A. Ogawa ${ }^{\dagger}$, V. L. Rykov ${ }^{\S 1}$ and N. Saito $\$ 2$ \\ ${ }^{\dagger}$ Penn State University, University Park, PA 16802, USA \\ $\S$ Wayne State University, Detroit, MI 48202, USA \\ $\ddagger$ RIKEN-BNL Research Center, Upton, NY 11973, USA
}

\begin{abstract}
The capabilities of RHIC with polarized protons to test the Lorentz structure of electroweak interactions and also the properties of MSSM Higgs, should it be discovered, are discussed.
\end{abstract}

\section{INTRODUCTION}

RHIC-Spin experiment is about being started. It has the solid program ${ }^{3}$, mainly concentrated around the measurements of proton spin dependent structure functions. For the last years, few attempts have been undertaken to also evaluate RHIC-Spin capabilities for testing physics beyond the Standard Model (SM) [1-3]. Since the recent suggestion [4] on a considerable increase of RHIC luminosity in $p p$ mode along with a sizeable energy increase, RHIC-Spin potential in this area became looking even more promising.

In this report, we update our earlier study [2] of RHIC-Spin capabilities to explore the Lorentz structure of electroweak interactions and also provide some estimates on whether Higgs sector could be reachable at RHIC. In our considerations, we assume $\sqrt{S}=500 \mathrm{GeV}$ and the luminosity of $0.8 \mathrm{fb}^{-1} /$ year for RHIC before an upgrade (RHIC-500), and $\sqrt{S}=650 \mathrm{GeV}$ and the luminosity of $\sim 20 \mathrm{fb}^{-1} /$ year for the after upgrade (RHIC-650).

1) Supported in part by the U.S. Department of Energy Grant DE-FG0292ER40713.

2) This work has been done partly within the framework of RIKEN RHIC-Spin project.

3) See plenary talk by N. Saito in this proceedings. 


\section{LORENTZ STRUCTURE OF QUARK ELECTROWEAK CURRENT}

Lorentz structure of electroweak interactions had always been and remains to be the focus of many precise nuclear and particle physics experiments. Over the last few years, the first measurements of the proton's weak magnetism have been accomplished [5]. Much attention had also been paid to test the universality of the electroweak interactions, in general, and to evaluating electroweak dipole moments of quarks and leptons, in particular. The current experimental constraints to magnetic and electric dipole moments of quarks and leptons, except electrons and muons, are still quite far from the values, predicted in the SM. If any of these moments were found to be nonzero and above SM's expectations, this would be a clear signal of a new physics beyond SM [6,7].

The most stringent experimental constraints, applicable to all components of quark and $\tau$-lepton dipole moments, come from the recent analyses $[6,7]$ of electroweak data from high energy colliders. In these analyses, it has been assumed that theories beyond the SM, emerging at some characteristic energy scale above $W / Z$ mass, have effects at low energies $E \leq M_{W, Z}$, and these effects can be taken into account by considering a Lagrangian that extends the SM Lagrangian, $L_{S M}$ : $L=L_{S M}+L_{\text {eff }}$. To preserve the consistency of the low energy theory, it had also been assumed that $L_{\text {eff }}$ is $\mathrm{SU}(3) \otimes \mathrm{SU}(2) \otimes \mathrm{U}(1)$ gauge invariant. Phenomenologically, this extension is equivalent to an introduction of a tensor coupling of fermions to gauge bosons which had also been discussed in Refs. $[8,2,3]$ :

$$
L_{\text {eff }}^{\text {charged }}=\frac{g}{2 \sqrt{2} \cdot \Lambda}\left\{\bar{q}_{d} \sigma^{\mu \nu}\left(f_{T}^{+}+f_{T}^{-} \gamma_{5}\right) q_{u} \partial_{\nu} W_{\mu}^{-}+\bar{q}_{u} \sigma^{\mu \nu}\left(f_{T}^{*+}-f_{T}^{*-} \gamma_{5}\right) q_{d} \partial_{\nu} W_{\mu}^{+}\right\}
$$

In Eq. (1), representing the charged-current part ${ }^{4}$ of $L_{e f f}, g$ is the electroweak coupling constant, $\Lambda$ is the energy scale of the "full strength" tensor interactions, and the asterisk denotes the complex conjugate. The notations $q_{u}$ and $q_{d}$ are for the "upper" $(u \text { and } c)^{5}$ and "lower" $(d, s, b)$ quarks, respectively. The CP- and $T$-invariance of model (1) is broken if any or all "formfactors" $f_{T}^{ \pm}$were complex 6 .

With the coupling (1), a number of prohibited in the SM spin asymmetries must show up in the annihilation of polarized $q \bar{q} \rightarrow W^{ \pm} / Z^{0} / \gamma \rightarrow l \bar{l}[2,3]$. For polarized proton collisions, particularly interesting would be single-spin asymmetries with transverse polarization, arising from the interference of SM's and tensor couplings, because: a) these asymmetries are strongly suppressed in SM and b) they are expected to have a good sensitivity to anomalous interactions due to quite strong correlations between the proton spin and polarizations of high- $x$ valence quarks, that participated in gauge boson production [9]. The triple-vector correlations,

4) With omitted quadratic terms, proportional to $g^{2} W_{\mu} W_{\nu}$; the neutral-current $L_{\text {eff }}$ looks similarly.

5) $t$-quark is virtually not reachable at RHIC.

6) In this paper: $\gamma_{5}=-i \gamma^{0} \gamma^{1} \gamma^{2} \gamma^{3}$ and $\sigma^{\mu \nu}=\frac{1}{2}\left(\gamma^{\mu} \gamma^{\nu}-\gamma^{\nu} \gamma^{\mu}\right)$. 
$\propto\left(\boldsymbol{k} \cdot\left[\boldsymbol{\zeta}_{\boldsymbol{q}}^{\perp} \times \boldsymbol{p}_{\boldsymbol{q}}\right]\right)$, give rise to the "left-right" asymmetry $A_{N}$, while nonzero "updown" asymmetry $A_{T}$ comes from $P$ - and $C P$-violating ${ }^{7}$ two-vector correlations, $\propto\left(\boldsymbol{\zeta}_{\boldsymbol{q}}^{\perp} \cdot \boldsymbol{k}\right)$. In the formulae above, $\boldsymbol{p}_{\boldsymbol{q}}$ and $\boldsymbol{\zeta}_{\boldsymbol{q}}^{\perp}$ are for the momentum and transverse polarization of an incident quark, and $\boldsymbol{k}$ is the momentum of a final lepton. The respective asymmetries $\hat{a}_{N, T}$ in $W^{ \pm}$production at the quark interaction level, integrated over the phase space of final leptons, would be as follows:

$$
\begin{aligned}
& \hat{a}_{N} \simeq \frac{3 \pi}{16} \cdot \frac{M_{W}}{\Lambda} \cdot \operatorname{Re}\left\{f_{T}^{+} \mp f_{T}^{-}\right\} /\left\{1+\frac{M_{W}^{2}}{4 \Lambda^{2}}\left(\left|f_{T}^{+}\right|^{2}+\left|f_{T}^{-}\right|^{2}\right)\right\} \\
& \hat{a}_{T} \simeq \frac{3 \pi}{16} \cdot \frac{M_{W}}{\Lambda} \cdot \operatorname{Im}\left\{f_{T}^{-} \mp f_{T}^{+}\right\} /\left\{1+\frac{M_{W}^{2}}{4 \Lambda^{2}}\left(\left|f_{T}^{+}\right|^{2}+\left|f_{T}^{-}\right|^{2}\right)\right\}
\end{aligned}
$$

Formulae $(2,3)$ have been obtained, using the solutions of Ref. [2] and with the assumptions that, the SM part of $q \bar{q} W$-interactions as well as the lepton coupling to $W^{ \pm}$were purely $V-A$. The upper and lower signs correspond to $W^{+}$and $W^{-}$ productions, respectively.

TABLE 1. RHIC-Spin sensitivity to $q \bar{q} W$ tensor coupling. Constraints $(2 \sigma)$ in columns $2-3$ are for the asymmetries at quark $(\hat{a})$ and proton $(A \simeq \hat{a} / 2[9,3])$ interaction levels. RHIC sensitivities $(2 \sigma)$ to $A_{N, T}$ are shown in columns $4-5$, assuming a $70 \%$ proton beam polarization.

\begin{tabular}{||c|c|c||c|c||}
\hline \multicolumn{3}{||c||}{ Constraints from Ref. [6] } & RHIC sensitivity in $\left(W^{+} / W^{-}\right)$modes \\
\hline$\left(\left|f_{T}^{+}\right|^{2}+\left|f_{T}^{-}\right|^{2}\right) \cdot M_{W}^{2} / \Lambda^{2}$ & $\hat{a}_{N, T}$ & $A_{N, T}$ & RHIC-500 & RHIC-650 \\
\hline$\leq 0.15$ & $\lesssim(20-30) \%$ & $\lesssim(10-15) \%$ & $\sim(1.5 / 3.0) \%$ & $\sim(0.2 / 0.4) \%$ \\
\hline
\end{tabular}

The experimental constraint on "formfactors" $f_{T}^{ \pm}$in charged current $u \leftrightarrow d$ transitions, converted to the convenient for our goals representation, is shown in Table 1, along with the respective limits on spin asymmetries in $p p^{\uparrow} \rightarrow W^{ \pm}+X \rightarrow$ $l \bar{l}+X$ processes. These limits then are compared with the RHIC sensitivities to $A_{N, T}$. One can observe that the current limits on $f_{T}$ in quark sector could be lowered by a factor of 5-10 at RHIC-500, and by almost an order of magnitude more after the upgrade to RHIC-650. And what is probably even more important, the real and imaginary parts of $f_{T}^{ \pm}$could be constrained individually from four independent measurements of $A_{N}$ and $A_{T}$ in $W^{+}$and $W^{-}$productions.

\section{WILL HIGGS PHYSICS BE REACHABLE AT RHIC?}

In the recent years, extensive efforts for cornering Higgs bosons resulted in the significantly shrunken area of the still allowed Higgs sector parameters. The actual discovery of Higgs particle(s) is expected to occur in not so remote future. Then, the focus will be shifted to studying their properties. Does RHIC with polarized protons have capabilities to contribute in this study?

7) In this particular model for $L_{\text {eff }}^{\text {charged }}$. 
The production cross section of Higgs strongly depends on the helicities of initial gluons and quarks. As a result, measurements of spin-correlations in polarized $p p$ collisions may allow us, for example, to determine $C P$-parities of Higgs states. Potentially, an interference between Higgs production and other SM processes in collisions of polarized gluons and quarks can generate a number of interesting singleand double-spin asymmetries ${ }^{8}$, including those sensitive to $C P$-violation in Higgs sector. In some cases, the polarization may help to improve signal-to-background ratio compared to unpolarized particle collisions.

Currently, the data favor low mass Higgs, probably just around $\sim 100 \mathrm{GeV}$, and for the Minimal Supersymmetric extension of the Standard Model (MSSM), the versions with large $\tan \beta \gtrsim 10$ seems as taking preferences ${ }^{9}$. Let's imagine for a moment that, just before RHIC-Spin upgrade, a neutral scalar ${ }^{10}$ has been discovered with the mass $M_{h} \sim 115-120 \mathrm{GeV}$, and its characteristics were consistent with the MSSM Higgs for $\tan \beta \simeq 30$. The estimated production cross section, $\sigma_{h}$, for such boson(s) at RHIC-650 would be $\sim 0.5$ pb with approximately equal contributions of $g g \rightarrow h$ and $q \bar{q} \rightarrow h$ subprocesses ${ }^{11}$. For an integrated luminosity of $20 \mathrm{fb} /$ year, one may expect a yield of $\sim 10^{4} \mathrm{~h} /$ year.

The main decay modes of low mass Higgs scalars are: $h \rightarrow b \bar{b}$ with the branching of $\sim 90 \%$, and $h \rightarrow \tau^{+} \tau^{-}$with the branching of $\sim 8-9 \%$. At the early stage, both these modes have been determined as hardly be suitable for the Higgs searches. However, as it was pointed out in Ref. [13], $h \rightarrow \tau^{+} \tau^{-}$decay might become useful "to provide confirming evidence for a signal found in other modes" ${ }^{12}$. In this report, as a first step toward understanding the Higgs-at-RHIC problem, we will start with the evaluation of only these two modes with the highest branchings, keeping in mind of course, that future studies could reveal better ways for the Higgs sector exploration at $\mathrm{RHIC}^{13}$.

The main obstacle for using $b \bar{b}$ channel at hadron colliders is the large QCD background from $g g \rightarrow b \bar{b}$. At RHIC-650, the estimated with PYTHIA production cross section $\sigma_{Q C D}^{b \bar{b}}$ for $b \bar{b}$-pairs in the mass interval of $115 \times(1 \pm 10 \%) \mathrm{GeV}$ is $\gtrsim 10^{3} \mathrm{pb}$. Resulting from cross section estimates Higgs signal-to-background ratio, $\sigma_{h} / \sigma_{Q C D}$, at $\lesssim 5 \cdot 10^{-4}$ does not seem too encouraging, particularly for studying Higgs properties. Potentially, an interference between $g g \stackrel{Q C D}{\rightarrow} b \bar{b}$ and $g g \stackrel{h}{\rightarrow} b \bar{b}$ could improve this ratio up to $\sim \sqrt{\sigma_{h} / \sigma_{Q C D}} \sim 10^{-2}$. Unfortunately, in the ultra-relativistic limit of massless $b$-quark, these two channels do not interfere. As a result, the actual interference term is additionally suppressed by a factor $m_{b} / M_{h} \sim 0.03-0.04$, which brings it back to the same low level of $\lesssim 5 \cdot 10^{-4}$.

8) Similar to the ones described, for example, in Refs. [10].

9) See, for example, $[11,12]$ and references therein.

10) Or scalars; for large $\tan \beta$, the theory allows for all three MSSM neutral bosons, $h^{0}, H^{0}$ and $A^{0}$, to be sitting simultaneously in the mass region from $\sim 100$ to $130 \mathrm{GeV}$ [12].

11) Notation $h$ is used here as a generic name for either $h^{0}, H^{0}$ or $A^{0}$.

12) See also Ref. [12], page 29 for the similar qualification.

13) See, for example, J. F. Gunion and T. C. Yan, Phys. Rev. Lett., 71 (1993) 488. 
The background to $\tau^{+} \tau^{-}$pairs from Higgs decays predominantly comes from the Drell-Yan process: $q \bar{q} \rightarrow \gamma / Z^{0} \rightarrow \tau^{+} \tau^{-}$. For RHIC-650, PYTHIA estimates the Drell-Yan cross section for $\tau^{+} \tau^{-}$at $\sim 0.25 \mathrm{pb}$ in the $\tau$-pair mass interval of $115 \times(1 \pm 10 \%) \mathrm{GeV}$. Taking into account $\sim 8 \%$ branching of Higgs to $\tau^{+} \tau^{-}$, the signal-to-background ratio in this mode would be $\sim 15 \%$. With the detection efficiency for high-mass $\tau$-pairs at about $20 \%$, the event rate in the mass interval above should be expected at $\sim 10^{3} \tau$-pairs/year. For this rate, $\sim 15 \%$ excess of events due to Higgs decays should be detectable well above the statistical fluctuations. Then, for example the $C P$-parity of a detected Higgs boson could be determined by measuring the sign of the contributing to the cross section doublespin correlation $\left(\zeta_{q}^{\perp} \cdot \zeta_{\bar{q}}^{\perp}\right)$ in collisions of transversely polarized protons. However, the statistics of just $\sim 10^{2}$ of $h \rightarrow \tau^{+} \tau^{-}$decays/year may not be sufficient for measuring double-spin asymmetries. Unfortunately, we cannot count on potentially more sensitive single-spin ones which could arise from an interference of two competing $q \bar{q} \rightarrow \tau^{+} \tau^{-}$channels. This interference will be vanishingly small because Drell-Yan pairs are mainly produced by light quarks, while the respective part of Higgs cross section will predominantly be due to $b \bar{b}$-annihilation.

\section{CONCLUSION}

It has been shown that RHIC with polarized protons will have highly competitive capabilities for hunting anomalies in the Lorentz structure of electroweak interaction due to physics beyond SM.

The low-mass Higgs might be reachable at high luminosity RHIC-650, although finding appropriate modes to study polarization phenomena in Higgs sector will be a quite challenging task.

Authors appreciate the valuable discussions with D. Boer, D. A. Cinabro, S. Dawson, R. L. Jaffe, J. S. Lange, T. Maruyama and J. Soffer. One of us (VLR) is thankful to T. M. Cormier for the support of his participation in SPIN2000 Symposium.

\section{REFERENCES}

1. P. Taxil and J.-M. Virey, Phys. Lett. B404 (1997) 302, B441 (1998) 376, Phys. Rev. D55 (1997) 4480; J. Murata, Report to the RHIC Spin Collaboration, Oct. 2000 (unpublished).

2. V. L. Rykov, Proc. of SPIN-98 Symposium (September 1998), p. 450-452; hep-ex/9908050.

3. S. Kovalenko, I. Schmidt, J. Soffer, hep-ph/9912529.

4. T. Roser, Private communications, and www.rhichome.bnl.gov/RHIC/luminosity/upgrade/.

5. B. Mueller et al. (SAMPLE Collaboration), Phys. Rev. Lett., 78 (1997) 3824.

6. R. Escribano, E. Masso, Nucl. Phys., B429 (1994) 19.

7. R. Escribano, E. Masso, Phys. Lett., B301 (1993) 419; Phys. Lett., B395 (1997) 369; T. G. Rizzo, Phys. Rev., D56 (1997) 3074; K. Ackerstaff et al. (OPAL Collaboration), Z. Phys., C74 (1997) 403; M. Acciarri et al. (L3 Collaboration), Phys. Lett., B426 (1998) 207; G. A. Gonzalez-Sprinberg, A. Santamaria, J. Vidal, hep-ph/0002203. 
8. G. L. Kane, G. A. Ladinsky and C.-P. Yuan, Phys. Rev., D45 (1992) 45.

9. J. Soffer, Nucl. Phys. (Proc. Suppl.), 64 (1998) 143.

10. E. Akawa et al., hep-ph/9912373; J. I. Illana, hep-ph/9912467, and references therein.

11. P. Igo-Kemenes in Rev. of Part. Phys., Eur. Phys. J., C15 (2000) 274; J. Erler, hep-ph/0010153; T. Affolder et al. (CDF Collaboration), hep-ex/0010052.

12. M. Carena, J. S. Conway, H. E. Harber, J. D. Hobbs, hep-ph/0010338.

13. J. F. Gunion, H. E. Haber, G. Kane and S. Dawson, The Higgs Hunter's Guide, AddisonWesley Publishing, 1990, p. 168. 